\title{
Molecular Signature of Tumors with Monoallelic 13q14 Deletion: a Case Series of Spindle Cell Lipoma and Genetically-Related Tumors Demonstrating a Link Between FOXO1 Status and p38 MAPK Pathway
}

\author{
Karina Uehara $^{1} \cdot$ Fukino Ikehara $^{1} \cdot$ Ryo Shibuya $^{2} \cdot$ Iwao Nakazato $^{3} \cdot$ Mariko Oshiro $^{4}$. \\ Masaya Kiyuna $^{5}$ - Yasuka Tanabe $^{1} \cdot$ Zensei Toyoda $^{1} \cdot$ Kiyoto Kurima $^{6}$. \\ Shinichiro Kina ${ }^{7} \cdot$ Masanori Hisaoka $^{2}$. Takao Kinjo ${ }^{1}$
}

Received: 23 May 2017 / Accepted: 1 September 2017 /Published online: 8 September 2017

(C) The Author(s) 2017. This article is an open access publication

\begin{abstract}
Spindle cell/pleomorphic lipomas (SCLs), cellular angiofibromas (CAFs) and mammary-type myofibroblastomas (MFBs) are rare benign mesenchymal tumors with monoallelic 13q14 deletion. They are predicted to have a common pathogenic mechanism due to shared similar histological and immunohistochemical features; however, pathological consequences of monoallelic 13 q14 deletion remain unknown. We previously reported a CAF case with monoallelic 13q14 deletion in which the tumor expressed decreased levels of FOXO1 and RB1, both of which were encoded in $13 \mathrm{q} 14$, and increased
\end{abstract}

Takao Kinjo

kinjotko@med.u-ryukyu.ac.jp

1 Division of Morphological Pathology, Department of Basic Laboratory Sciences, School of Health Sciences, University of the Ryukyus, 207 Uehara, Nishihara, Okinawa 903-0215, Japan

2 Department of Pathology and Oncology, School of Medicine, University of Occupational and Environmental Health, Fukuoka, Japan

3 Department of Pathology, Okinawa Prefectural Nanbu Medical Center and Children's Medical Center, Okinawa, Japan

4 Health Information Management Major, Management and Information Science Division, Faculty of International Studies, Meio University, Okinawa, Japan

5 Department of Pathology, Tomishiro Chuo Hospital, Okinawa, Japan

6 Department of Regenerative Medicine, Graduate School of Medicine, University of the Ryukyus, Okinawa, Japan

7 Department of Oral and Maxillofacial Functional Rehabilitation, Graduate School of Medicine, University of the Ryukyus, Okinawa, Japan reactive oxygen species (ROS) levels. We further demonstrated the activation of $\mathrm{p} 38$ mitogen-activated protein kinase (p38 MAPK) pathway induced by oxidative stress. We hypothesized that SCLs, CAFs and MFBs would share common molecular signatures involving FOXO1, ROS and p38 MAPK and that their expression patterns were different from those tumors without monoallelic $13 \mathrm{q} 14$ deletion such as solitary fibrous tumors (SFTs). We compared the expression levels of FOXO1, RB1, ROS markers and several signal transduction factors between SCLs and SFTs. SCLs expressed decreased levels of FOXO1 and RB1, whereas SFTs showed no change. Both tumor types exhibited increased markers of ROS; however, nuclear localization of phosphorylated p38 was significantly more frequent in SCLs than that in SFTs, suggesting p38 MAPK activation by oxidative stress. SFTs showed lower p38 MAPK activity and higher $\beta$ catenin expression, implying that oxidative stress was caused by increased cellular proliferation stress. Finally, CAFs and MFBs showed changes similar to those observed in SCLs. Overall, tumors with monoallelic 13q14 deletion showed shared molecular signatures that might be associated with pathogenesis.

Keywords Monoallelic 13q14 deletion · RB 1 - FOXO 1 . Reactive oxygen species · p38 MAPK
Abbreviations
SCL Spindle cell/pleomorphic lipoma
CAF Cellular angiofibroma
MFB Mammary-type myofibroblastoma
SFT Solitary fibrous tumor 
AA Aggressive angiomyxoma

DFSP Dermatofibrosarcoma protuberans

WLS Well-differentiated liposarcoma

MLS Myxoid liposarcoma

PLS Pleomorphic liposarcoma

\section{Introduction}

Spindle cell/pleomorphic lipomas (SCLs), cellular angiofibromas (CAFs) and mammary-type myofibroblastomas (MFBs) are rare benign mesenchymal tumors with monoallelic 13q14 deletion [1-3]. These tumors share similar histopathological and immunohistochemical features; however, molecular events induced by this genetic alteration remain unclear. Two tumor suppressor genes, RB1 and FOXO1, are located in 13q14. While RB1 is a well-established tumor suppressor, FOXO family of transcription factors are associated with diverse functions such as cell cycle regulation, differentiation, apoptosis, DNA repair and reactive oxygen species (ROS) detoxification [4-6]. Specifically, FOXO1 was shown to be associated with alveolar rhabdomyosarcoma as a result of chromosome alteration and is considered as a tumor suppressor [4].

In a previous study, we reported a CAF case with monoallelic $13 \mathrm{q} 14$ deletion. The tumor expressed decreased levels of FOXO1 and RB1 as well as increased levels of oxidative stress markers, 8-hydroxy-2'deoxyguanosine (8-OHdG) and 4-hydroxy-2-nonenal (4HNE). Furthermore, the p38 mitogen-activated protein kinase (p38 MAPK) pathway, which is often induced by ROS, was activated in that CAF case [7].

While the molecular mechanism underlying tumorigenesis associated with monoallelic 13q14 deletion is not known, based on these findings, we hypothesized that SCLs, CAFs and MFBs would share common molecular signatures involving FOXO1 expression, ROS status and p38 MAPK activity. To examine our hypothesis, we conducted a series of comparative studies between tumors with monoallelic 13q14 deletion, SCLs, CAFs and MFBs, and those without 13q14 deletion, such as solitary fibrous tumors (SFTs), aggressive angiomyxomas (AAs), dermatofibrosarcoma protuberans (DFSP), welldifferentiated liposarcoma (WLS), myxoid liposarcoma (MLS) and pleomorphic liposarcoma (PLS) which are frequently considered in differential diagnosis. We analyzed clinicopathological characteristics and performed immunohistochemistry to assess the expression levels of FOXO1, RB1 and oxidative stress markers, the p38 MAPK activity and other signal transduction pathways associated with pathogenesis.

\section{Methods}

\section{Case Selection}

Representative hematoxylin and eosin-stained slides of cases were reviewed for case selection. The majority of cases were derived from the consultation files of one of the authors (M. H.), which included 52 SCLs, 3 CAFs, 3 MFBs, 36 SFTs and 2 AAs. In addition, 3 SCLs, 1 CAF, 4 SFTs, 2 AAs, 11 DFSP, 12 WLS, 10 MLS and 4 PLS were retrieved from the surgical pathology files of other institutions. Several aspects of clinical, histological, immunohistochemical and cytogenetic information on a CAF sample were previously reported [7]. Since the SFT harbor inversion of chromosome $12 \mathrm{q} 13$ resulting in NAB2-STAT6 fusion gene, detection of STAT6 by immunohistochemistry has become a diagnostic tool for SFT. In present study, $91 \%(32 / 35)$ of SFT were positive for STAT6. The study protocol was approved by the Institutional Review Boards of University of the Ryukyus, Okinawa, Japan (No. 791, June 2, 2015) and University of Occupational and Environmental Health, Fukuoka, Japan (H27-029, June 23, 2015). All procedures performed in this study were carried out in accordance with the Declaration of Helsinki.

\section{Histopathological Analysis}

The tumors were processed, stained with hematoxylin and eosin and were analyzed using immunohistochemistry. Primary antibodies used in this study were $\alpha$-smooth muscle actin (Dako, Glostrup, Denmark), CD34 (Dako), desmin (Dako), Ki-67 (Dako), S-100 (Dako), RB1 (Abcam, Cambridge, United Kingdom), p16 (Abcam), STAT6 (Santa Cruz, Dallas, Texas), FOXO1 (Cell Signaling Technology, Boston, Massachusetts), phosphorylated p38 (Cell Signaling Technology), $\beta$-catenin (Millipore, Billerica, Massachusetts), 8-OHdG or 4-HNE (both from Japan Institute for the Control of Aging, Shizuoka, Japan), and the details of the staining methods were described in the previous report [7]. The appropriate positive and negative controls were included in each staining. The immunoreactivity and positive extent of the markers were semiquantitatively scored using a similar method as that reported by Sangoi et al. [8]. In brief, the immunoreactivity was graded as negative, weak, moderate, or strong. The percentage of tumor cells with each marker was semiquantitatively scored as $0(0 \%-9 \%), 1+(10 \%-49 \%), 2+$ $(50 \%-89 \%)$ and $3+(\geq 90 \%)$. For each antibody, the immunoreactivity of more than weak and the percentage score of tumor cells more than $1+$ were judged as positive. Only nuclear positive signals for RB1, FOXO1, 8-OHdG, p16, phosphorylated $\mathrm{p} 38$ and $\beta$-catenin were used for scoring. 


\section{Fluorescence In Situ Hybridization Analysis}

Fluorescence in situ hybridization (FISH) analysis was performed using a chromosome 13 (13q14)-specific probe (Poseidon $^{\mathrm{TM}}$ repeat-free FISH probes, Kreatech Diagnostics, Amsterdam, The Netherlands), which include RB1 gene but not FOXO1 gene, according to the manufacturer's instructions and as described previously [7].

\section{Statistical Analysis}

Data were analyzed by two-tail Mann-Whitney U-test. A $P$ value of $<0.05$ was considered statistically significant for all analyses.

\section{Results}

\section{Clinical Findings of Tumors with Monoallelic 13q14 Deletion}

The summary of clinicopathological parameters of tumors with monoallelic 13q14 are shown in Table 1. The SCLs affected 45 males and 8 females; two cases were unknown. The median age was 62 years (range, $21-87$ years). The most common affected sites were the head and neck, followed by the trunk. The CAFs affected 3 males and 1 female, and the median age was 53.5 years (range, 49-69 years). The CAF from female was in the vagina, whereas the CAFs from the males were in the groin, testis and scrotum. The MFBs affected 1 male and 2 females, with a median age of 64 years (range, 54-65 years). The MFBs from the females were in the groin and knee, whereas the MFB from the male was in the mammary gland.

\section{FISH for 13q14}

All SCLs were examined by FISH for $13 q 14$. Among a total of 55 SCLs, monoallelic 13q14 deletion was confirmed in 43 cases $(78 \%)$, and 12 cases unsuitable for FISH or negative for monoallelic $13 \mathrm{q} 14$ deletion were excluded for subsequent analyses.

\section{RB1 and FOXO1 Expression in Tumors with or Without Monoallelic 13q14 Deletion}

RB1 and FOXO1 expression was evaluated because these genes are encoded in 13q14 region. Nuclear RB1 expression was confirmed in 29 of $42(69 \%)$ SCLs; however, the majority exhibited either weak or moderate immunoreactivity. RB1 expression was also detected in 1 of $4 \mathrm{CAFs}$ and all MFBs (3/3). SFTs showed higher rates of positive RB1 expression $(32 / 40 ; 80 \%)$ than those observed in SCLs, and the immunoreactivity to RB1 in SFTs tended to be higher than that observed in SCLs. Finally, PLSs (0/4) showed no expression and WLSs (3/11) demonstrated low frequency, whereas the others including AAs (3/4), DFSPs (5/11) and MLSs (8/10) were positive for RB1 (Table 2, Fig. 1).

Nuclear FOXO1 expression was detected in 16 of 30 (53\%) SCLs. The majority of FOXO1-positive cases exhibited weak immunoreactivity. FOXO1 was expressed in 1 of 4 CAFs, and in all the MMF specimens analyzed; however, none of those showed a strong FOXO1 immunoreactivity. The majority of SFTs $(31 / 36: 86 \%)$ were positive for FOXO1, and FOXO1 immunoreactivity tended to be higher than that observed in SCLs. Although PLSs (0/4) showed no expression, AAs (2/4), DFSPs (5/11), WLSs (9/11) and MLSs $(9 / 10)$ expressed FOXO1 (Table 2, Fig. 1).

\section{Oxidative Stress Markers in Tumors with or Without Monoallelic 13q14 Deletion}

Two oxidative stress markers were evaluated in this study. The majority of SCLs were positive for both 8 -OHdG (36/42: $86 \%)$ and 4-HNE (34/42: $81 \%)$. The majority of CAFs and MFBs also exhibited positive staining for these two markers. Similarly, SFTs also showed increased expression of 8-OHdG (31/40: 78\%) and 4-HNE (34/40: 85\%). Finally, most of the AAs, DFSPs, WLSs, MLSs and PLSs indicated high positivity for both markers (Table 2, Fig. 2).

\section{p38 MAPK and $\beta$-Catenin Signaling in Tumors with or Without Monoallelic 13q14 Deletion}

Nuclear phosphorylated p38 localization was demonstrated in 28 of 42 (67\%) SCLs. The majority of those positive for phosphorylated p38 showed moderate immunoreactivity. A positive nuclear phosphorylated $\mathrm{p} 38$ was also seen in all 4 CAFs, as well as 2 out of 3 MMFs. Of a total 40 SFTs, 15 cases showed nuclear phosphorylated p38 localization (15/40; $38 \%$ ); however, the extent and immunoreactivity of phosphorylated p38 in SFTs tended to be lower than those observed in SCLs. Finally, most of AAs and MLSs were positive for nuclear phosphorylated p38, whereas DFSPs, WLSs and PLSs showed low positive rate (Table 2, Fig. 3).

Because p16 is one of the important signal transduction factors downstream from $\mathrm{p} 38$ MAPK pathway, we compared expression of $\mathrm{p} 16$ between SCLs and SFTs. All SCLs expressed p16 (42/42: 100\%), and among them, 38 cases were either moderately or strongly immunoreactive for $\mathrm{p} 16$. Of SFTs, 76\% (29/38) were positive for p16, whereas p16 immunoreactivity varied across specimens (Table 2, Fig. 3 ).

Nuclear $\beta$-catenin localization was observed in 15 of 42 (36\%) SCLs; the $\beta$-catenin immunoreactivity was either moderate or weak in these cases. No nuclear $\beta$-catenin was detected in CAFs, whereas 1 out of 3 MMFs expressed nuclear $\beta$ - 
Table 1 Summary of clinicopathological features of specimens analyzed in this study

\begin{tabular}{|c|c|c|c|c|c|c|c|c|c|}
\hline & SCL & $\mathrm{CAF}$ & MFB & AA & SFT & DFSP & WLS & MLS & PLS \\
\hline \multicolumn{10}{|l|}{ Sex } \\
\hline Male & 45 & 3 & 1 & 1 & 24 & 7 & 8 & 5 & 1 \\
\hline Female & 8 & 1 & 2 & 3 & 16 & 5 & 4 & 5 & 3 \\
\hline \multicolumn{10}{|l|}{ Age(y) } \\
\hline$<50$ & 11 & 1 & 0 & 2 & 13 & 8 & 2 & 5 & 0 \\
\hline$\geqq 50$ & 42 & 3 & 3 & 2 & 27 & 4 & 10 & 5 & 4 \\
\hline Median & 62.0 & 53.5 & 64.0 & 44.0 & 58.5 & 44 & 58.5 & 49.5 & 66 \\
\hline Range & $21-87$ & $49-69$ & $54-65$ & $28-57$ & $9-84$ & $3-68$ & $40-79$ & $33-81$ & $61-92$ \\
\hline \multicolumn{10}{|l|}{ Site } \\
\hline Head and neck & 24 & 0 & 0 & 0 & 11 & 2 & 0 & 0 & 1 \\
\hline Shoulder & 10 & 0 & 0 & 0 & 2 & 1 & 1 & 0 & 0 \\
\hline Chest wall & 1 & 0 & 0 & 0 & 2 & 2 & 0 & 0 & 0 \\
\hline Pleura & 0 & 0 & 0 & 0 & 6 & 0 & 0 & 0 & 0 \\
\hline Mammary gland & 0 & 0 & 1 & 0 & 0 & 0 & 0 & 0 & 0 \\
\hline Abdominal wall & 0 & 0 & 0 & 0 & 1 & 1 & 0 & 0 & 0 \\
\hline Lung & 0 & 0 & 0 & 0 & 2 & 0 & 1 & 0 & 0 \\
\hline Retroperitoneum & 0 & 0 & 0 & 0 & 5 & 0 & 2 & 0 & 0 \\
\hline Kidney & 0 & 0 & 0 & 0 & 2 & 0 & 1 & 0 & 0 \\
\hline Pelvic cavity & 0 & 0 & 0 & 0 & 2 & 0 & 0 & 0 & 0 \\
\hline Back & 8 & 0 & 0 & 0 & 1 & 3 & 0 & 0 & 0 \\
\hline Buttock & 0 & 0 & 0 & 0 & 2 & 0 & 0 & 2 & 0 \\
\hline Extremities & 11 & 0 & 1 & 0 & 1 & 3 & 7 & 8 & 3 \\
\hline Genital region & 1 & 4 & 1 & 4 & 1 & 0 & 0 & 0 & 0 \\
\hline Etc & 0 & 0 & 0 & 0 & 2 & 0 & 0 & 0 & 0 \\
\hline
\end{tabular}

$S C L$, spindle cell/pleomorphic lipoma; $C A F$, cellular angiofibroma; $M F B$, myofibroblastoma; $S F T$, solitary fibrous tumor; $A A$, aggressive angiomyxoma; $D F S P$, dermatofibrosarcoma protuberans; $W L S$, well-differentiated liposarcoma; $M L S$, myxoid liposarcoma; $P L S$, pleomorphic liposarcoma catenin. In contrast, the majority of SFTs (28/40: 70\%) were positive for $\beta$-catenin which tended to be expressed stronger than that observed in SCLs (Table 2, Fig. 3).

\section{Discussion}

SCLs, CAFs and MFBs share a common genetic defect: monoallelic 13q14 deletion [1-3]. While two tumor suppressor genes, RB1 and FOXO1, are encoded in this region, the precise molecular pathogenesis of these tumors is unknown. To date, there are relatively few reports investigating the pathogenesis of these tumors. Chen et al. reported that soft tissue tumors with monoallelic $13 \mathrm{q}$ deletions, such as SCLs and CAFs as well as the majority of MMFs did not express RB1, which might lead to subsequent tumor development. Although the mechanism underlying the decreased level of RB1 expression is yet unclear, they suggested a mutation on the remaining allele or an epigenetic change that affects the expression of RB1 [9]. Magro et al. reported deletion of FOXO1 in mammary- and vaginal- type MFBs by FISH analysis and proposed that tumors with 13 q14 deletion belong to a single entity with a continuous spectrum of different morphological presentations [10]. However, FOXO1 expression level in these tumors has not been reported. In the present study, we compared the expression of RB1 and FOXO1 between SCLs (tumor with monoallelic 13q14 deletion) and SFTs (that without 13q14 deletion), because the number of cases is sufficient for statistical analysis between these tumors. Contrary to our prediction, the rate of RB1 positivity was not different between SCLs and SFTs, however, the extent of positive signal and immunoreactivity to RB1 in SCLs were lesser than those in SFTs. Because we used 13q14 FISH probe which included RB1 region, one signal in the present FISH experiment suggested loss of one RB1 gene. Therefore, the extent and immunoreactivity of RB1 in SCLs may reflect the gene dosage of $\mathrm{RB} 1$, i.e., one allele of RB1 gene by monoallelic deletion. The difference of RB1 positivity between present study and the study by Chen et al. (2012) could have been due to the different anti-RB1 antibody used. The positive rate as well as both positive extent and 
Table 2 Summary of immunohistochemical results of tumor specimens analyzed in this study

\begin{tabular}{|c|c|c|c|c|c|c|c|c|c|}
\hline & SCL & CAF & MFB & SFT & AA & DFSP & MLS & WDLS & PLS \\
\hline \multicolumn{10}{|l|}{ RB1 } \\
\hline - Positive rate & $29 / 42$ & $1 / 4$ & $3 / 3$ & $32 / 40$ & $3 / 4$ & $5 / 11$ & $8 / 10$ & $3 / 11$ & $0 / 4$ \\
\hline $\begin{array}{l}\cdot \text { Positive extent } \\
3+\end{array}$ & 1 & 0 & 1 & 21 & 1 & 0 & 0 & 0 & 0 \\
\hline $2+$ & 6 & 0 & 1 & 8 & 2 & 0 & 0 & 0 & 0 \\
\hline $1+$ & 22 & 1 & 1 & 3 & 0 & 5 & 8 & 3 & 0 \\
\hline 0 & 13 & 3 & 0 & 8 & 1 & 6 & 2 & 8 & 0 \\
\hline \multicolumn{10}{|c|}{ • Immunoreactivity } \\
\hline Strong & 2 & 0 & 0 & 8 & 0 & 0 & 1 & 0 & 0 \\
\hline Moderate & 14 & 2 & 2 & 18 & 2 & 1 & 3 & 4 & 0 \\
\hline Weak & 19 & 1 & 1 & 8 & 2 & 5 & 5 & 4 & 0 \\
\hline None & 7 & 1 & 0 & 6 & 0 & 5 & 1 & 3 & 4 \\
\hline \multicolumn{10}{|l|}{ FOXO1 } \\
\hline - Positive rate & $16 / 30$ & $1 / 4$ & $3 / 3$ & $31 / 36$ & $2 / 4$ & $5 / 11$ & $9 / 10$ & $9 / 11$ & $0 / 4$ \\
\hline \multicolumn{10}{|l|}{ - Positive extent } \\
\hline $3+$ & 1 & 0 & 0 & 10 & 1 & 0 & 7 & 3 & 0 \\
\hline $2+$ & 4 & 0 & 1 & 16 & 1 & 1 & 1 & 3 & 0 \\
\hline $1+$ & 11 & 1 & 2 & 5 & 0 & 4 & 1 & 3 & 0 \\
\hline 0 & 14 & 3 & 0 & 5 & 2 & 6 & 1 & 2 & 4 \\
\hline \multicolumn{10}{|c|}{ - Immunoreactivity } \\
\hline Strong & 1 & 0 & 0 & 10 & 0 & 0 & 7 & 3 & 1 \\
\hline Moderate & 4 & 1 & 1 & 14 & 2 & 5 & 3 & 2 & 0 \\
\hline Weak & 12 & 1 & 2 & 7 & 1 & 1 & 0 & 5 & 1 \\
\hline None & 13 & 2 & 0 & 5 & 1 & 5 & 0 & 1 & 2 \\
\hline \multicolumn{10}{|l|}{ 8-OHdG } \\
\hline $\begin{array}{l}\text { - Positive rate } \\
\text { - Positive extent }\end{array}$ & \multicolumn{8}{|c|}{ - Positive extent } & $4 / 4$ \\
\hline $3+$ & 24 & 4 & 1 & 15 & 1 & 1 & 1 & 0 & 1 \\
\hline $2+$ & 6 & 0 & 1 & 10 & 1 & 0 & 2 & 4 & 0 \\
\hline $1+$ & 6 & 0 & 0 & 6 & 0 & 5 & 4 & 1 & 3 \\
\hline 0 & 6 & 0 & 1 & 9 & 2 & 1 & 3 & 6 & 0 \\
\hline \multicolumn{10}{|c|}{ • Immunoreactivity } \\
\hline Strong & 7 & 1 & 1 & 0 & 0 & 5 & 4 & 8 & 3 \\
\hline Moderate & 18 & 3 & 0 & 21 & 2 & 1 & 1 & 1 & 0 \\
\hline Weak & 12 & 0 & 2 & 12 & 1 & 0 & 3 & 2 & 0 \\
\hline None & 5 & 0 & 0 & 7 & 1 & 1 & 2 & 0 & 1 \\
\hline \multicolumn{10}{|l|}{ 4-HNE } \\
\hline $\begin{array}{l}\text { - Positive rate } \\
\text { - Positive extent }\end{array}$ & \multicolumn{8}{|c|}{ - Positive extent } & $4 / 4$ \\
\hline $3+$ & 27 & 2 & 1 & 27 & 0 & 2 & 0 & 2 & 1 \\
\hline $2+$ & 4 & 2 & 1 & 1 & 2 & 0 & 1 & 4 & 0 \\
\hline $1+$ & 3 & 0 & 0 & 6 & 1 & 1 & 7 & 4 & 3 \\
\hline 0 & 8 & 0 & 1 & 6 & 1 & 1 & 2 & 2 & 0 \\
\hline \multicolumn{10}{|c|}{ • Immunoreactivity } \\
\hline Strong & 6 & 2 & 1 & 7 & 0 & 1 & 5 & 9 & 2 \\
\hline Moderate & 14 & 1 & 0 & 20 & 1 & 1 & 3 & 2 & 1 \\
\hline Weak & 14 & 1 & 2 & 7 & 2 & 1 & 0 & 1 & 1 \\
\hline None & 8 & 0 & 0 & 6 & 0 & 1 & 2 & 0 & 0 \\
\hline \multicolumn{10}{|c|}{ phosphorylated p38 } \\
\hline $\begin{array}{l}\text { - Positive rate } \\
\text { - Positive extent }\end{array}$ & $28 / 42$ & $4 / 4$ & $2 / 3$ & $15 / 40$ & $3 / 4$ & $3 / 10$ & $6 / 10$ & $2 / 12$ & $0 / 4$ \\
\hline $3+$ & 14 & 4 & 1 & 0 & 0 & 0 & 0 & 1 & 0 \\
\hline $2+$ & 10 & 0 & 1 & 3 & 2 & 0 & 2 & 0 & 0 \\
\hline $1+$ & 4 & 0 & 0 & 12 & 1 & 3 & 4 & 1 & 0 \\
\hline 0 & 14 & 0 & 1 & 25 & 1 & 7 & 4 & 10 & 4 \\
\hline - Immunoreactiv & & & & & & & & & \\
\hline Strong & 2 & 1 & 1 & 0 & 0 & 4 & 5 & 3 & 3 \\
\hline Moderate & 19 & 2 & 1 & 2 & 3 & 1 & 2 & 6 & 0 \\
\hline Weak & 7 & 1 & 0 & 14 & 1 & 0 & 2 & 3 & 0 \\
\hline None & 14 & 0 & 1 & 24 & 0 & 5 & 1 & 0 & 1 \\
\hline p16 & & & & & & & & & \\
\hline $\begin{array}{l}\text { - Positive rate } \\
\text { - Positive extent }\end{array}$ & $42 / 42$ & $4 / 4$ & $3 / 3$ & $29 / 38$ & $3 / 4$ & $6 / 6$ & $3 / 10$ & $3 / 6$ & \\
\hline $3+$ & 22 & 3 & 2 & 8 & 1 & 3 & 0 & 0 & ND \\
\hline $2+$ & 15 & 1 & 1 & 12 & 2 & 2 & 1 & 0 & ND \\
\hline $1+$ & 5 & 0 & 0 & 9 & 0 & 1 & 2 & 3 & ND \\
\hline 0 & 0 & 0 & 0 & 9 & 1 & 0 & 7 & 3 & ND \\
\hline
\end{tabular}


Table 2 (continued)

\begin{tabular}{|c|c|c|c|c|c|c|c|c|c|}
\hline & SCL & $\mathrm{CAF}$ & MFB & SFT & AA & DFSP & MLS & WDLS & PLS \\
\hline \multicolumn{10}{|c|}{ • Immunoreactivity } \\
\hline Strong & 23 & 2 & 2 & 7 & 0 & 6 & 0 & 0 & ND \\
\hline Moderate & 15 & 1 & 0 & 12 & 3 & 0 & 3 & 0 & ND \\
\hline Weak & 4 & 1 & 1 & 12 & 1 & 0 & 1 & 3 & ND \\
\hline None & 0 & 0 & 0 & 7 & 0 & 0 & 6 & 3 & ND \\
\hline \multicolumn{10}{|l|}{$\beta$-catenin } \\
\hline - Positive rate & $15 / 42$ & $0 / 4$ & $1 / 3$ & $28 / 40$ & $3 / 4$ & & & & \\
\hline \multicolumn{10}{|l|}{ - Positive extent } \\
\hline $3+$ & 0 & 0 & 1 & 18 & 1 & ND & ND & ND & ND \\
\hline $2+$ & 4 & 0 & 0 & 7 & 2 & ND & ND & ND & ND \\
\hline $1+$ & 11 & 0 & 0 & 3 & 0 & ND & ND & ND & ND \\
\hline 0 & 27 & 4 & 2 & 12 & 1 & ND & ND & ND & ND \\
\hline \multicolumn{10}{|c|}{ - Immunoreactivity } \\
\hline Strong & 0 & 0 & 0 & 11 & 0 & ND & ND & ND & ND \\
\hline Moderate & 9 & 1 & 1 & 12 & 2 & ND & ND & ND & ND \\
\hline Weak & 10 & 0 & 0 & 5 & 1 & ND & ND & ND & ND \\
\hline None & 23 & 3 & 2 & 12 & 1 & ND & ND & ND & ND \\
\hline
\end{tabular}

$S C L$, spindle cell/pleomorphic lipoma; $C A F$, cellular angiofibroma; $M F B$, myofibroblastoma; $S F T$, solitary fibrous tumor; $A A$, aggressive angiomyxoma; $D F S P$, dermatofibrosarcoma protuberans; $W L S$, well-differentiated liposarcoma; $M L S$, myxoid liposarcoma; $P L S$, pleomorphic liposarcoma. SCL and SFT were highlighted by bold text for comparison

immunoreactivity to FOXO1 in SCLs were lower than those in SFTs $(53 \%$ versus $86 \%, p<0.05)$. Because the FISH probe used in the present study did not include FOXO1 region, and FOXO1 gene is approximately $7,8 \mathrm{Mb}$ apart from RB1 gene, the monoallelic $13 \mathrm{q} 14$ deletion did not necessarily demonstrate the loss of one allele of FOXO1 gene. The expression control of FOXO1 may have a similar expression mechanism of FOXP2, one of FOX gene family, and its mutation is associated with a neurodevelopmental defect in acquisition of spoken language. FOXP2 undergoes random monoallelic expression (RMAE), which results in three distinct expression statuses: expression of "both alleles" in some cells and expression of either the maternal or paternal allele in other cells. FOXP2 expression of "both alleles" is controlled under cis-regulatory element that map to $3 \mathrm{Mb}$ away from the FOXP2 gene locus. Therefore, a deletion of the cis-regulatory element, which is demonstrated in a patient with developmental verbal dyspraxia, results in FOXP2 haploinsufficiency (monoallelic expression) [11]. Because cis-regulatory element of FOXO1 in $13 \mathrm{q} 14$ is unknown and whether FOXO1 operates RMAE is yet unclear, further study regarding the mechanism of FOXO1 expression is required to understand the impact of monoallelic $13 \mathrm{q} 14$ deletion. The present study suggested that monoallelic 13 q14 deletion in SCLs affects the expression of both RB1 and FOXO1. The number of CAFs and MFBs were limited in our study; however, both CAFs and MFBs showed low expression levels of RB1 and FOXO1. Based on the downregulation of both these tumor suppressors, we hypothesize that these events may be contributing to a shared pathogenic mechanism of tumors with monoallelic 13q14 deletions, as proposed by Magro et al. [10].

In a previous study, we reported a CAF patient with monoallelic 13q14 deletion and loss of FOXO1 expression, which was accompanied by increased expression of oxidative stress markers [7]. We also showed the activation of p38 MAPK pathway, which is often induced by oxidative stress $[12,13]$. The present study confirmed the increased oxidative stress and subsequent p38 MAPK activation in SCLs. These findings suggested that the loss of FOXO1 function in tumors with monoallelic 13q14 deletion induces oxidative stress, leading to the activation of p38 MAPK signaling. The role of p38 MAPK signaling in soft tissue tumors remains controversial. Two groups reported that therapy using SRC family kinase inhibitors, or artesunate, antimalarial drug, induced growth inhibition of rhabdomyosarcoma through the activation of p38 MAPK $[14,15]$, whereas another group reported that p38 MAPK might play a role in induction of human telomerase reverse transcriptase in sarcomas, suggesting an association between p38 MAPK and malignant transformation [16].

In this study, we found higher p38 MAPK activity in SCLs than that in SFTs $(67 \%$ versus $38 \%, p<0.05)$. While the rate and intensity of oxidative stress marker expression were similar between SCLs and SFTs, the responses to oxidative stress might differ between them. Given the low rate of FOXO1 positivity in SCLs, the response to oxidative stress might be impaired, resulting in increased ROS levels that lead to p38 MAPK activation. Since p16 is one of the important signal transduction factors downstream from p38 MAPK pathway, the high rate of p16 positivity in SCLs also suggested p38 


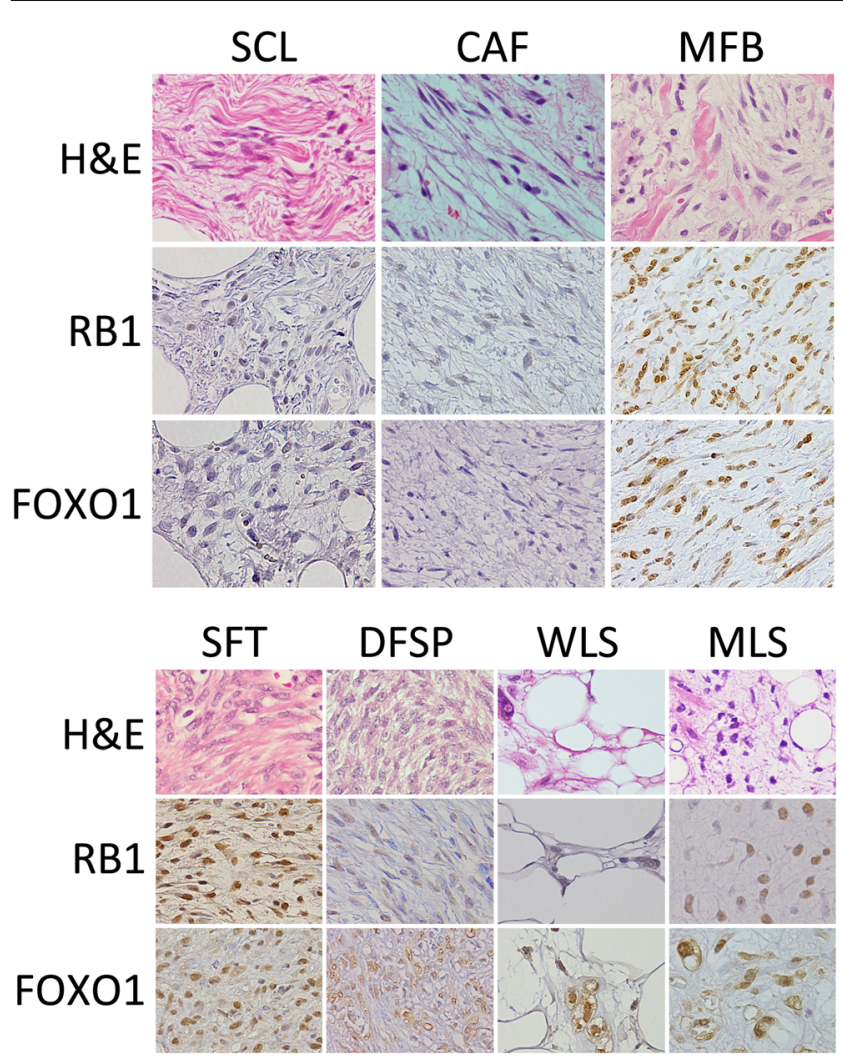

Fig. 1 Representative images of tumor specimens stained for hematoxylin and eosin, immunohistochemically stained for RB1 and FOXO1 are shown. SCL: spindle cell/pleomorphic lipoma, CAF: cellular angiofibroma, MFB: myofibroblastoma, SFT: solitary fibrous tumor, DFSP: dermatofibrosarcoma protuberans, WLS: well-differentiated liposarcoma, MLS: myxoid liposarcoma. SCL and CAF showed decreased expression of RB1 and FOXO1, whereas other tumors exhibited various expression levels of either marker

MAPK activation. CAFs and MFBs showed similar results to those in SCLs. These findings suggested that tumors with monoallelic 13q14 deletion express decreased FOXO1 levels, leading to increased ROS accumulation that result in the activation of p38 MAPK pathway.

Despite similar levels of ROS between SCLs and SFTs, FOXO1 positivity rate was higher in SFTs than SCLs, suggesting that the response to ROS elicited by FOXO1 in SFTs is intact. In addition, p38 MAPK activity was lower in SFTs than SCLs; again, these results might reflect an intact FOXO1 mediated antioxidant response in SFTs. In general, oxidative stress in cancer cells is aggravated by oncogene expression, increased metabolic activity, hypoxic conditions, chronic inflammation and damaged mitochondrial function [17-19]. As neither oxidative stress nor p38 MAPK activity in SFTs has been reported to date, a literature search did not result in any information on the antioxidant response in SFT; however, the present study suggests that increased oxidative stress in SFTs induces normal FOXO1 activation.

Hoogeboom et al. reported that binding of FOXO family members to $\beta$-catenin inhibits $\beta$-catenin/T cell factor (TCF) transcriptional activity, ultimately resulting in suppression of the Wnt signaling [20], implicating FOXO as tumor suppressor. Therefore, we analyzed nuclear $\beta$-catenin translocation and hypothesized that tumor with monoallelic $13 \mathrm{q} 14$ deletion would show increased nuclear $\beta$-catenin translocation. However, in contrast to our hypothesis, $\beta$-catenin expression and its nuclear localization were lower in SCLs than SFTs (36\% versus $70 \%$ ). These results implied that normal functions of adenomatous polyposis coli (APC) were preserved in SCLs, which lead to proper proteasomal degradation of $\beta$ catenin. Based on these results, we proposed that the $\mathrm{Wnt} / \beta$ catenin pathway is not related to the pathogenesis in tumors with monoallelic 13q14 deletion.

In the present study, the number of CAF and MFB cases was few, therefore, the comparison of positivity of each marker between these tumors and SCLs showed limited correlation. Furthermore, the present immunohistochemical analysis demonstrated that $\mathrm{p} 38$ MAPK activation was induced by increased ROS, but the resulting biological effects such as cell proliferation, apoptosis and cell senescence remain unclear. Further molecular analyses using fresh specimen is needed.

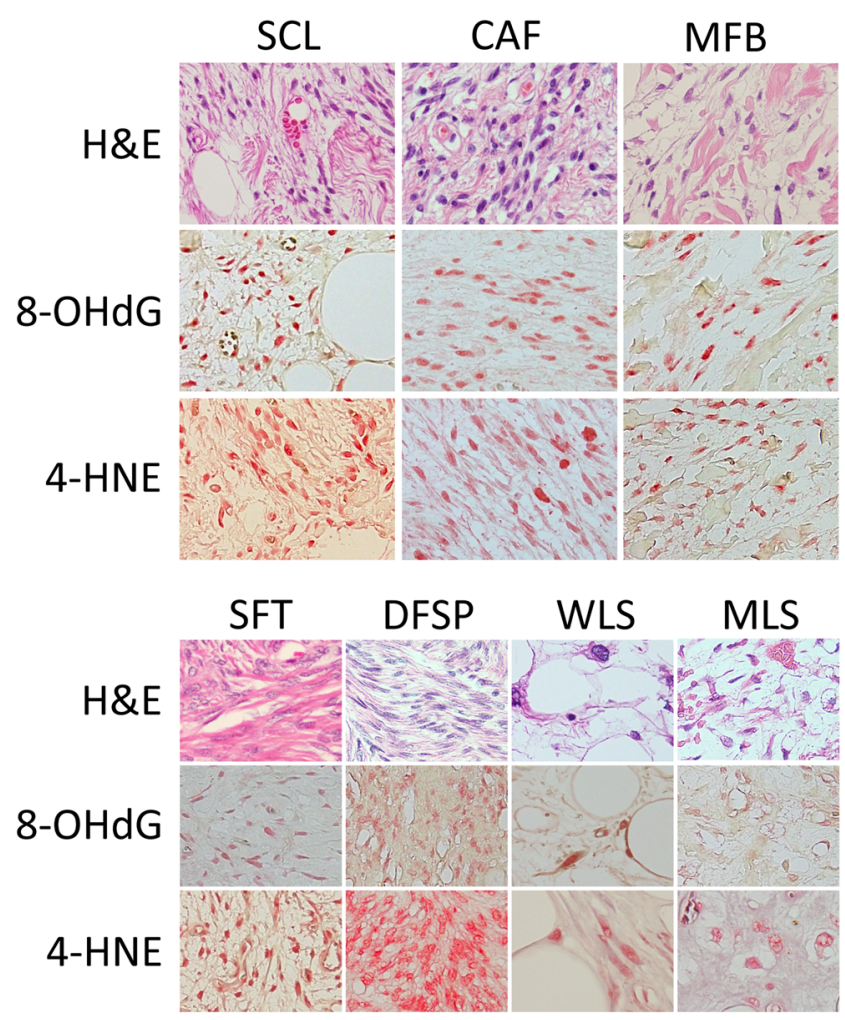

Fig. 2 Representative images of tumor specimens stained for hematoxylin and eosin, immunohistochemically stained for 8-hydroxy2'-deoxyguanosine (8-OHdG) and 4-hydroxy-2-nonenal (4-HNE) are shown. SCL: spindle cell/pleomorphic lipoma, CAF: cellular angiofibroma, MFB: myofibroblastoma, SFT: solitary fibrous tumor, DFSP: dermatofibrosarcoma protuberans, WLS: well-differentiated liposarcoma, MLS: myxoid liposarcoma. Specimens from tumors with monoallelic deletion of $13 \mathrm{q} 14$ as well as those without $13 \mathrm{q} 14$ deletion demonstrated increased expression of markers of oxidative stress 


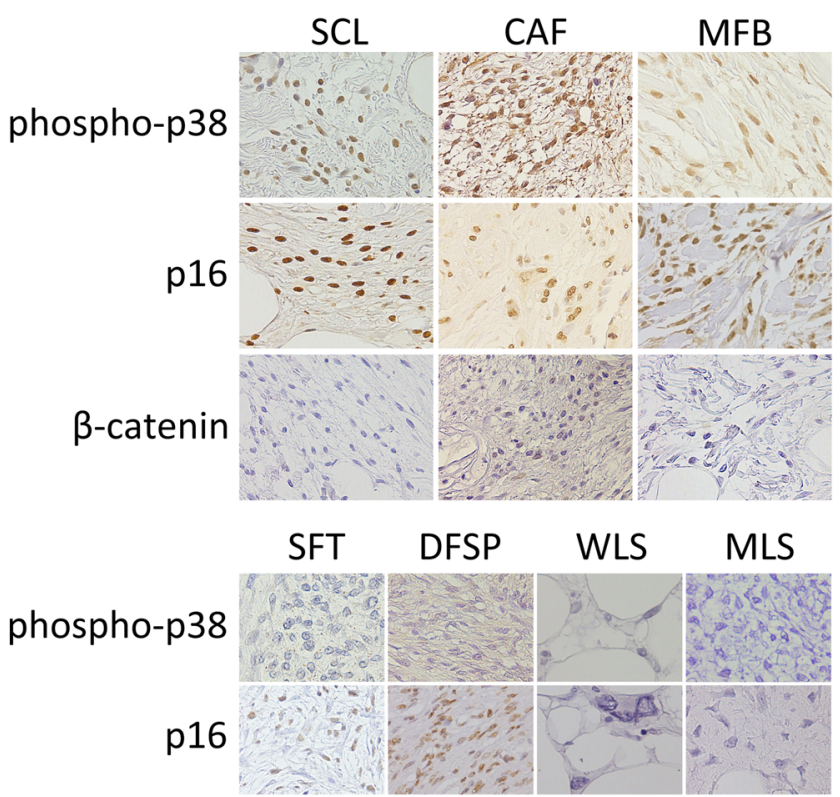

Fig. 3 Representative images of tumor specimens immunohistochemically stained for phosphorylated p38, p16 and $\beta$-catenin are shown. SCL: spindle cell/pleomorphic lipoma, CAF: cellular angiofibroma, MFB: myofibroblastoma, SFT: solitary fibrous tumor, DFSP: dermatofibrosarcoma protuberans, WLS: well-differentiated liposarcoma, MLS: myxoid liposarcoma. SCL, CAF and MFB specimens were positive for phosphorylated $\mathrm{p} 38$ and p16 in the nucleus and showed decreased expression of $\beta$ catenin. In contrast, SFT specimens exhibited decreased phosphorylated p38 and p16 expression levels. The others showed various expression levels of these markers

In summary, the tumors with monoallelic 13q14 deletion show decreased expression level of FOXO1, which is encoded in the $13 \mathrm{q} 14$ region, and increased levels of oxidative stress markers as well as higher p38 MAPK activity. The above results lead us to hypothesize that those tumors with monoallelic $13 q 14$ deletion share molecular signatures involving the increase in the oxidative stress markers that are induced by decreased FOXO1 expression; furthermore, increased oxidative stress result in the activation of p38 MAPK pathway. The present study suggests these molecular events are associated with tumorigenesis caused by $13 \mathrm{q} 14$ deletion.

Acknowledgements We thank Mr. Yoshihide Oshiro (University of the Ryukyus) for his technical assistance.

\section{Compliance with Ethical Standards}

Conflict of Interest Statement The authors declare that they have no conflict of interest.

Ethical Approval All procedures performed in studies involving human participants were in accordance with the ethical standards of the institutional research committees and with the 1964 Helsinki declaration and its later amendments or comparable ethical standards. For this type of study formal consent is not required.

Open Access This article is distributed under the terms of the Creative Commons Attribution 4.0 International License (http:// creativecommons.org/licenses/by/4.0/), which permits unrestricted use, distribution, and reproduction in any medium, provided you give appropriate credit to the original author(s) and the source, provide a link to the Creative Commons license, and indicate if changes were made.

\section{References}

1. Flucke U, van Krieken JH, Mentzel T (2011) Cellular angiofibroma: analysis of 25 cases emphasizing its relationship to spindle cell lipoma and mammary-type myofibroblastoma. Mod Pathol 24:82-89

2. Fritchie KJ, Carver P, Sun Y, Batiouchko G, Billings SD, Rubin BP, Tubbs RR, Goldblum JR (2012) Solitary fibrous tumor: is there a molecular relationship with cellular angiofibroma, spindle cell lipoma, and mammary-type myofibroblastoma? Am J Clin Pathol 137:963-970

3. Hameed M, Clarke K, Amer HZ, Mahmet K, Aisner S (2007) Cellular angiofibroma is genetically similar to spindle cell lipoma: a case report. Cancer Genet Cytogenet 177:131-134

4. Greer EL, Brunet A (2005) FOXO transcription factors at the interface between longevity and tumor suppression. Oncogene 24:7410-7425

5. Huang H, Tindall DJ (2007) Dynamic FoxO transcription factors. J Cell Sci 120:2479-2487

6. Maiese K, Chong ZZ, Shang YC, Hou J (2009) A "FOXO" in sight: targeting Foxo proteins from conception to cancer. Med Res Rev 29:395-418

7. Arakaki K, Chinen K, Kamiya M, Tanabe Y, Tawata N, Ikehara F, Uehara K, Shimabukuro H, Kinjo T (2014) Evidence for an association between increased oxidative stress and derangement of FOXO1 signaling in tumorigenesis of a cellular angiofibroma with monoallelic 13q14: a case report. Int J Clin Exp Pathol 7:8972-8979

8. Sangoi AR, Dulai MS, Beck AH, Brat DJ, Vogel H (2009) Distinguishing chordoid meningiomas from their histologic mimics: an immunohistochemical evaluation. Am J Surg Pathol 33:669-681

9. Chen BJ, Marino-Enriquez A, Fletcher CD, Hornick JL (2012) Loss of retinoblastoma protein expression in spindle cell/ pleomorphic lipomas and cytogenetically related tumors: an immunohistochemical study with diagnostic implications. Am J Surg Pathol 36:1119-1128

10. Magro G, Righi A, Casorzo L, Antonietta T, Salvatorelli L, Kacerovska D, Kazakov D, Michal M (2012) Mammary and vaginal myofibroblastomas are genetically related lesions: fluorescence in situ hybridization analysis shows deletion of $13 \mathrm{q} 14$ region. Hum Pathol 43:1887-1893

11. Adegbola AA, Cox GF, Bradshaw EM, Hafler DA, Gimelbrant A, Chess A (2015) Monoallelic expression of the human FOXP2 speech gene. Proc Natl Acad Sci U S A 112:6848-6854

12. Wang X, Liu JZ, Hu JX, Wu H, Li YL, Chen HL, Bai H, Hai CX (2011) ROS-activated p38 MAPK/ERK-Akt cascade plays a central role in palmitic acid-stimulated hepatocyte proliferation. Free Radic Biol Med 51:539-551

13. Torres M, Forman HJ (2003) Redox signaling and the MAP kinase pathways. Biofactors 17:287-296

14. Beccafico S, Morozzi G, Marchetti MC, Riccardi C, Sidoni A, Donato R, Sorci G (2015) Artesunate induces ROS- and p38 MAPK-mediated apoptosis and counteracts tumor growth in vivo in embryonal rhabdomyosarcoma cells. Carcinogenesis 36:1071-1083 
15. Casini N, Forte IM, Mastrogiovanni G, Pentimalli F, Angelucci A, Festuccia C, Tomei V, Ceccherini E, Di Marzo D, Schenone S, Botta M, Giordano A, Indovina P (2015) SRC family kinase (SFK) inhibition reduces rhabdomyosarcoma cell growth in vitro and in vivo and triggers $\mathrm{p} 38$ MAP kinase-mediated differentiation. Oncotarget 6:12421-12435

16. Matsuo T, Shimose S, Kubo T, Fujimori J, Yasunaga Y, Sugita T, Ochi M (2012) Correlation between p38 mitogen-activated protein kinase and human telomerase reverse transcriptase in sarcomas. $\mathrm{J}$ Exp Clin Cancer Res 31:5
17. Weinberg F, Chandel NS (2009) Reactive oxygen speciesdependent signaling regulates cancer. Cell Mol Life Sci 66: 3663-3673

18. Tong L, Chuang CC, Wu S, Zuo L (2015) Reactive oxygen species in redox cancer therapy. Cancer Lett 367:18-25

19. Tsuneki M, Nakamura Y, Kinjo T, Nakanishi R, Arakawa H (2015) Mieap suppresses murine intestinal tumor via its mitochondrial quality control. Sci Rep 5:12472

20. Hoogeboom D, Essers MA, Polderman PE, Voets E, Smits LM, Burgering BM (2008) Interaction of FOXO with betacatenin inhibits beta-catenin/T cell factor activity. J Biol Chem 283:9224-9230 